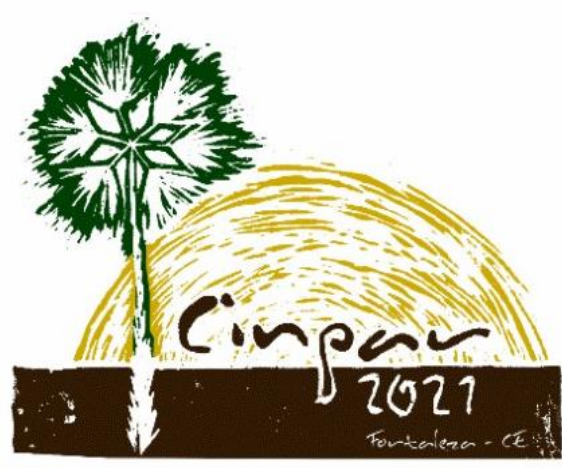

XVII Congresso Internacional sobre Patologia e Reabilitação das Construções

XVII Congreso Internacional sobre Patología y Rehabilitación de las Construcciones

XVII International Conference on Pathology and Constructions Rehabilitation

FORTALEZA (Brasil), 3 a 5 de junho de 2021

https://doi.org/10.4322/CINPAR.2021.098

\title{
Aplicação do software EMMA na análise do empacotamento máximo de partículas utilizando o Metacaulim.
}

\section{Application of EMMA software in the analysis of the maximum particle packaging using Metakaolin.}

\author{
B.A.S. Raul, N.C. P. Manoel, M.B.N. Vanessa ,A.F.M. Gilber, L.G.L. Alyne, H.P.F. Luis \\ CISNE, Quixadá/CE, Brasil, rai.rrs.pbranca@gmail.com \\ CISNE, Quixadá/CE, Brasil, cristianpereirapc241200@gmail.com \\ CISNE, Quixadá/CE, Brasil, vanessabn2000@gmail.com \\ CISNE, Quixadá/CE, Brasil,gilbermesquita11@gmail.com \\ CISNE, Quixadá/CE, Brasil,alynegomes345@gmail.com \\ CISNE, Quixadá/CE, Brasil, luis.franca@faculdadecisne.edu.br
}

\begin{abstract}
Resumo: Com a evolução do concreto e o aumento da sua aplicação em diferentes ambientes submetidos a diferentes temperaturas e intempéries da natureza, seu desempenho, durabilidade, e sua microestrutura vem sendo estudados para melhorar suas propriedades físicas e mecânicas. Para tornar as estruturas de concreto mais leves, sustentáveis e duráveis surgiram novos desafios na tecnologia dos materiais. Para que as propriedades mecânicas pudessem aumentar e, consequentemente, prover maior resistência e segurança para as obras, tais desafios precisam ser superados. Atualmente, na produção, aplicação e cura de concretos além da escolha dos materiais e da definição do traço adequado é necessário reduzir o número de vazios entre os grãos por meio do máximo empacotamento de partículas, proporcionando um ótimo índice de DTP (Distribuição do Tamanho de Partículas). O presente trabalho simulou traços de concreto utilizando o software EMMA para a mistura, usando como materiais o cimento CP ARI, areia e como material cimentício secundário para o concreto, o metacaulim, por contribuir no desempenho mecânico e durabilidade. Foram feitos testes utilizando valores diferentes para o valor de q (coeficiente de empacotamento), foram eles $q=0.25, q=0.30$ e $q=0.37$. $O$ que melhor se ajustou ao modelo modificado de Andreassen, parâmetro usado pelo programa, foi $q=0.25$ pela mínima distância adquirida entre a linha de parâmetro do EMMA e a linha gerada pela simulação.
\end{abstract}

Palavras-chave: Concreto, EMMA, Empacotamento, Metacaulim

\begin{abstract}
With the evolution of concrete and the increase in its application in different environments subjected to different temperatures and weather in nature, its performance, durability, and its microstructure have been studied to improve its physical and mechanical properties. To make concrete structures lighter, more sustainable and more durable, new challenges have arisen in the technology of materials. In order for the mechanical properties to increase and, consequently, provide greater resistance and safety for the works, such challenges need to be overcome. Currently, in the production, application and
\end{abstract}


curing of concrete, in addition to the choice of materials and the definition of the appropriate trace, it is necessary to reduce the number of voids between the grains by means of maximum particle packing, providing an excellent index of DTP (Size Distribution of Particles). The present work simulated concrete traces using the EMMA software for the mixture, using CP ARI cement, sand and as a secondary cement material for concrete, metakaolin, for contributing to the mechanical performance and durability. Tests were carried out using different values for the value of $q$ (packaging coefficient), they were $q=0.25, q=0.30$ and $q=0.37$. What best fitted the modified Andreassen model, a parameter used by the program, was $q=0.25$ due to the minimum distance acquired between the EMMA parameter line and the line generated by the simulation.

Keywords: Concrete, EMMA, Packaging, Metakaolin

\section{Introdução}

A tecnologia do concreto, modulada pela competitividade do mercado, vem buscando soluções para que o material atenda aos requisitos satisfatórios, baixa permeabilidade à água, redução na formação de estrias e alta resistência. Muito tem sido trabalhado no âmbito científico, pesquisas estas fomentadas por soluções para que as propriedades do concreto tragam segurança para a sua empregabilidade.

De acordo com METHA \& MONTEIRO (1994), os requisitos mais importantes de desempenho são a trabalhabilidade do concreto no estado fresco e a resistência do concreto no estado endurecido. A trabalhabilidade que é responsável pela facilidade com que a mistura pode ser lançada, compactada e acabada; e a resistência, que quando atingida conforme a necessidade está relacionada à durabilidade do concreto.

A composição do concreto, pode ser definida como a reação advinda da mistura do cimento, água, agregados miúdos, graúdos e aditivos químicos. Logo, permite-se adquirir grande resistência à compressão e à flexão. O comportamento dos materiais que envolvem a composição, está devidamente interligado com as interações entre as suas partículas. Desta maneira, o projeto da distribuição do tamanho das partículas é de suma importância, pois promove o empacotamento, agente que define as propriedades reológicas do material durante a mistura e no estado fresco. Quando o concreto no estado fresco possui características adequadas para a sua trabalhabilidade, como a consistência, atende de forma flexível às demandas da obra, podendo ser adequadamente compactado entre os diversos tipos de armaduras.

A noção de trabalhabilidade é, portanto, muito mais subjetiva que física. O componente físico mais importante da trabalhabilidade é a consistência, termo que, aplicado ao concreto, traduz propriedades intrínsecas da mistura fresca relacionadas com a mobilidade da massa e a coesão entre os elementos componentes, tendo em vista a uniformidade e a compacidade do concreto e o bom rendimento da execução. (BAUER, 2012).

De acordo com a NBR 7211 (2009), agregado para concreto deve ser "composto por grãos de minerais duros, compactos, duráveis, estáveis, limpos e que não interfiram no endurecimento e hidratação do cimento e também na proteção contra corrosão da armadura". Desta forma, devido a importância da escolha dos agregados para a mistura, vários são os testes para garantir o atendimento às especificações, como o de abatimento de tronco de cone (Slump Test) e o ensaio de resistência à compressão de corpos de prova. 
A distribuição granulométrica ideal pode ser obtida por modelos consagrados, como os propostos por Furnas, Andreassen e Alfred (CASTRO; PANDOLFELLI, 2009). Atualmente tornaram-se mais práticos e eficientes com a utilização de recursos computacionais, processo este, detalhado nas próximas etapas.

O principal objetivo será avaliar traços de concreto usando o software EMMA, gerando composições com os melhores empacotamentos de partícula, fator que contribuirá para a melhoria das propriedades do concreto estudado. $O$ primeiro passo consistirá na escolha dos materiais que irão compor o traço. Em seguida, deveremos considerar a mescla de densidade e quantidade dos materiais, formulando simulações, para que enfim, possamos ter as escolhas que trarão a melhor densidade de empacotamento de partículas. O software disponibilizará uma curva modelo que servirá de base para a construção do gráfico de DTP (Distribuição do Tamanho de Partícula).

Desta maneira, o traço resultou da interação entre os agregados, buscando gerar o melhor empacotamento, mediado pela escolha do seu modelo mais adequado e pelas características mais pertinentes. Para obter um concreto adequado para a sua utilização deve-se analisar as propriedades dos materiais que o compõe, proporcionalmente correto, assim como, a execução da mistura e o controle do concreto durante o estado fresco e endurecido (AMBROZEWICZ, 2012).

\section{Empacotamento de partículas}

O primeiro tratado sobre empacotamento foi publicado por Féret em 1892 e, desde então, diversos modelos têm sido propostos como ferramenta para calcular a densidade de empacotamento das partículas e, assim, otimizar misturas granulares de concretos, e essas misturas são, o cimento CPV ARI, areia e o metacaulim.

O estudo do empacotamento de partículas pode ser definido como o problema da correta seleção da proporção e do tamanho adequado dos materiais particulados, de forma que os vazios maiores sejam preenchidos por partículas menores, cujos vazios serão novamente preenchidos com partículas ainda menores e assim sucessivamente (OLIVEIRA et al., 2000).

Seus modelos são apresentados por equações matemáticas que descrevem como partículas de diferentes tamanhos que irão interagir geometricamente, desse modo a formação das partículas em definição de uma lei, $\mathrm{CPFT}=100$ (Dp/DI)q. Esses modelos calculam a densidade de empacotamento teórica de uma mistura baseada na distribuição granulométrica e na densidade de empacotamento de um determinado grupo de partículas. Dentre esses modelos disponíveis na literatura, o modelo de Alfred que também é conhecido como modelo de Andreasen, que foi modificado e proposto por FUNK e DINGER, se destaca na dosagem de concretos por possuir uma maior eficiência de empacotamento e ser mais ajustado com os sistemas particulados reais, no modelo onde o coeficiente e $q=0,37$.

O empacotamento de partículas permite uma evolução nas propriedades do concreto, promove um benefício ambiental por meio da utilização de resíduos e subprodutos industriais, promove também redução de emissões poluentes e economia de energia, mediante a redução do consumo de cimento na mistura. 


\section{Metodologia}

Definir traços para o concreto antes da sua aplicação, garante máxima eficiência e uma maior segurança ao se trabalhar com o mesmo, para isso, é necessário simulações para prever seu comportamento granular. No programa EMMA analyser (Elkem Materials Mixture Analyser) é possível construir milhares de distribuições de tamanho de partículas de materiais diferentes, ao manipular a distribuição do tamanho das partículas e, consequentemente prever a fluidez da mistura.

Os concretos podem ser feitos de acordo com uma DTP(Distribuição do tamanho de partícula) predeterminada ou alguma mistura de bom desempenho pode ser repetida com outras matérias-primas. Se uma fração moída por algum motivo mudou suas características, é relativamente simples ajustar a mistura para que a DTP desejada seja mantida. Ele analisa através de gráficos e tabelas a granulometria dos materiais individualmente e após a mistura dos mesmos. Para esse referido trabalho de pesquisa foi simulado um traço usando como materiais essenciais o Cimento CP Ari e como derivados Areia Média, tais materiais já tem seus dados de distribuição granulométrica inseridos na biblioteca do Software, além de uma gama de outros já existentes no banco de dados do EMMA.

Quadro 1 - Está presente a densidade e quantidade dos materiais, esta é alterada de acordo com o que se deseja utilizar no traço, aquela já é fornecida pelo software

\begin{tabular}{|c|c|c|c|}
\hline Material & Densidade $\left(\mathbf{k g} / \mathbf{m}^{\mathbf{3}}\right.$ ) & Quantidade (kg) & Vol\% \\
\hline Cimento CP Ari & 3,10 & 820 & 38,21 \\
\hline Areia Média & 2,30 & 800 & 50,24 \\
\hline Metacaulim & 2,50 & 200 & 11,55 \\
\hline
\end{tabular}

Após a adição dos materiais e a definição do traço, é necessário definir o modelo o qual servirá de referência para o empacotamento de partículas, o programa fornece o que melhor se encaixa para o máximo empacotamento de partículas, o modelo modificado de Andreassen.

Logo abaixo estão as curvas granulométricas dos 3 materiais usados, tais curvas foram geradas no software Excel, facilitando para que se tenha uma análise mais precisa com relação a distribuição granulométrica. Na figura 1 está representada a curva granulométrica da areia média, em seguida a figura 2 , a curva granulométrica do cimento CP ARI.

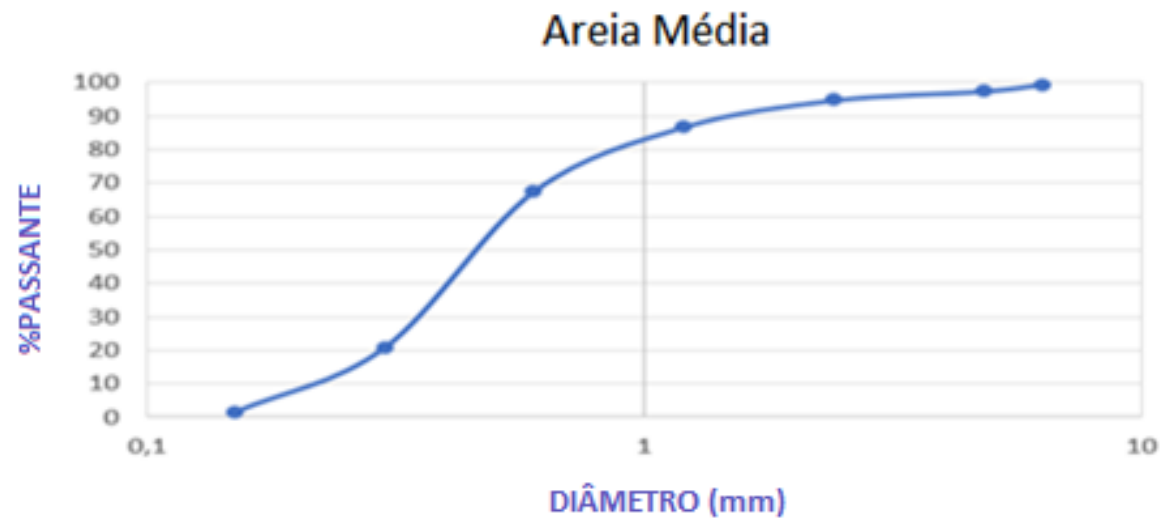

Figura 1 - Curva granulométrica da areia

Aplicação do software EMMA na análise do empacotamento máximo de partículas utilizando o Metacaulim. 


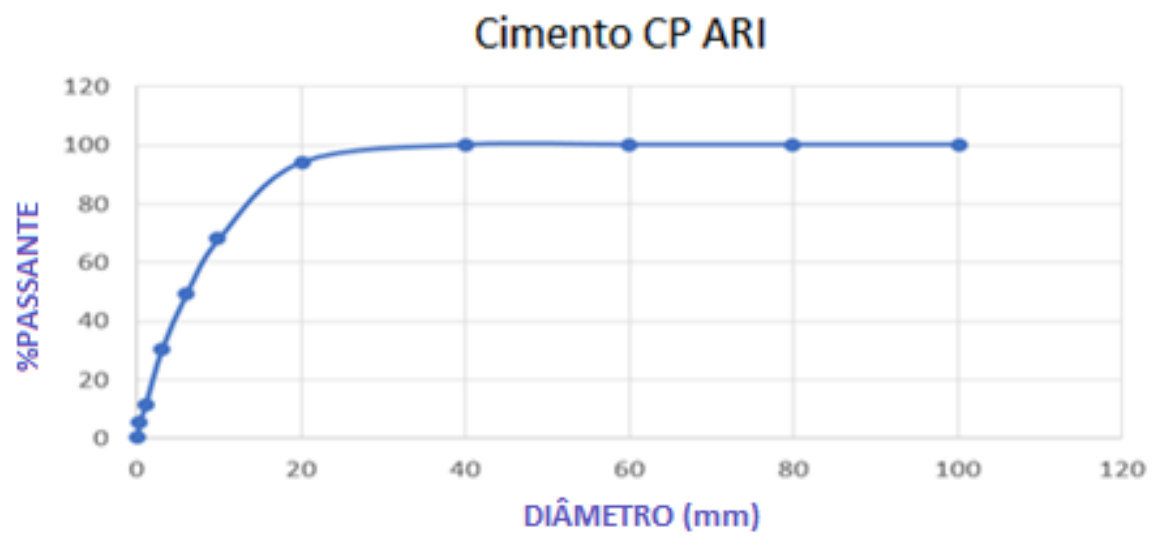

Figura 2 - Curva granulométrica do cimento CP Ari

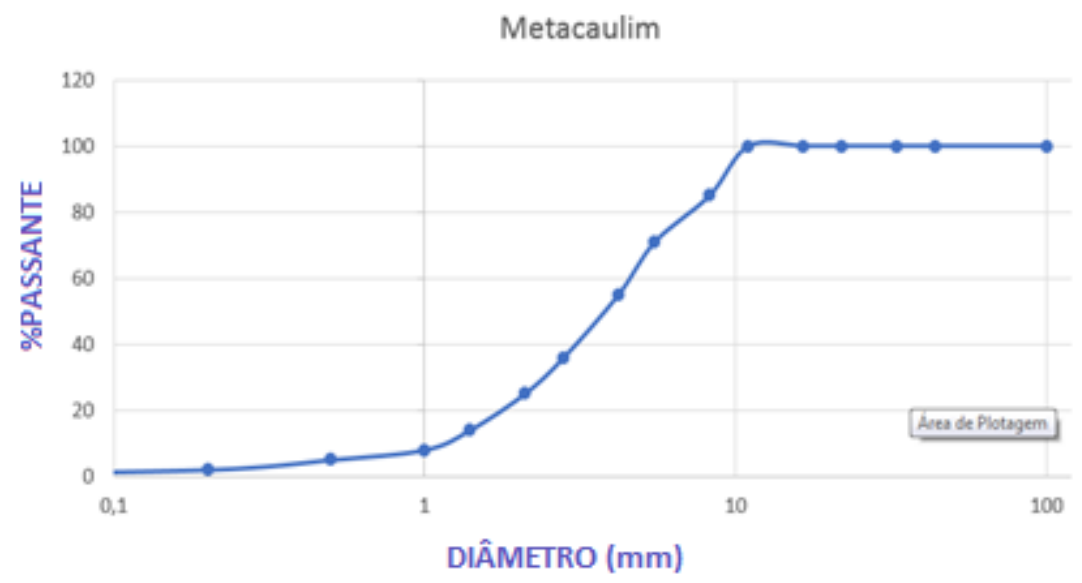

Figura 3 - Curva granulométrica do Metacaulim

\section{Resultados e discussões}

O concreto é um dos produtos com maior consumo no mundo, segundo a Federação Iberoamericana do Concreto Pré-misturado, em 2015, foi produzido mudialmente cerca de 2,4 bilhões de metros cúbicos de concreto pré-misturado. Sua correta produção beneficia para que durante sua aplicação e envelhecimento, possa haver uma evolução nas suas características mecânicas, para isso, diminuir os vazios é essencial. A adoção do valor correto do coeficiente de empacotamento $(q)$ contribui para uma melhor DTP.

Para a simulação do traço estudado foi usado três valores do coeficiente $q$ usando o modelo modificado de Andreassen, aonde por um gráfico de DTP é possível visualizar uma comparação entre a linha vermelha usada como parâmetro pelo modelo, e a linha azul que representa a mistura dos três materiais. No eixo Y temos a porcentagem do que passou do material e no eixo $\mathrm{X}$ temos o tamanho de partícula. 


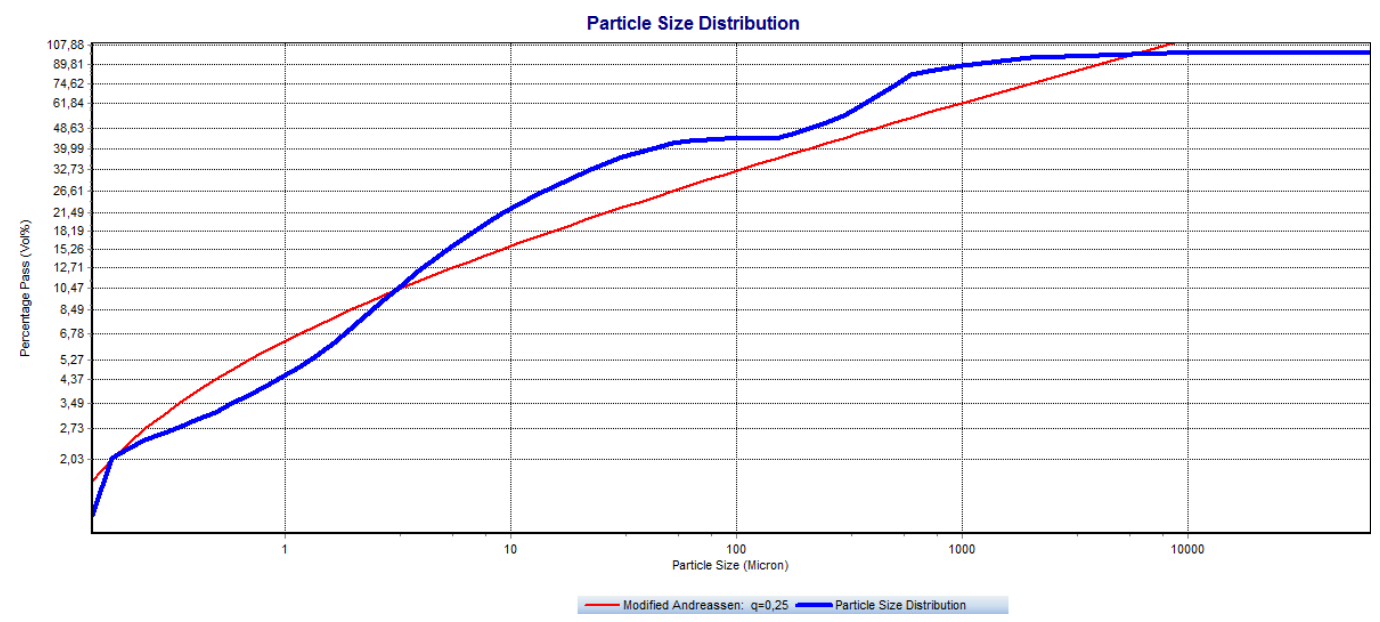

Figura 4 - Curva granulométrica do traço estudado, formulado com q=0,25

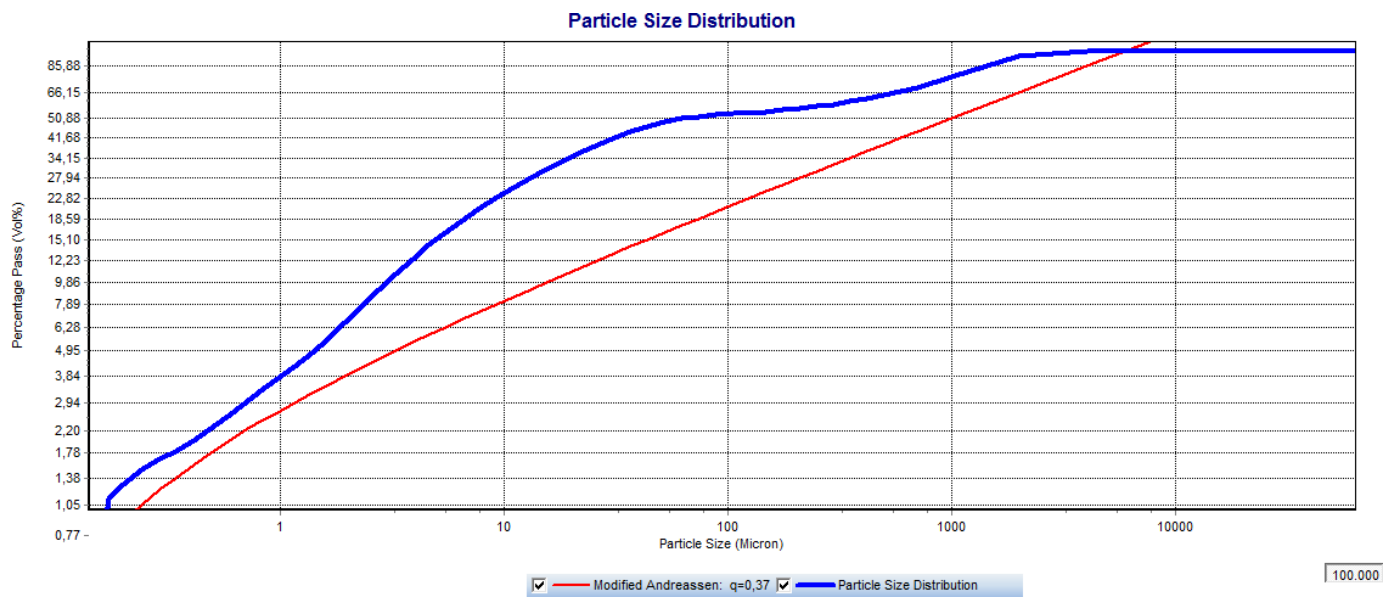

Figura 5 - Curva granulométrica do traço estudado, formulado com q=0,30

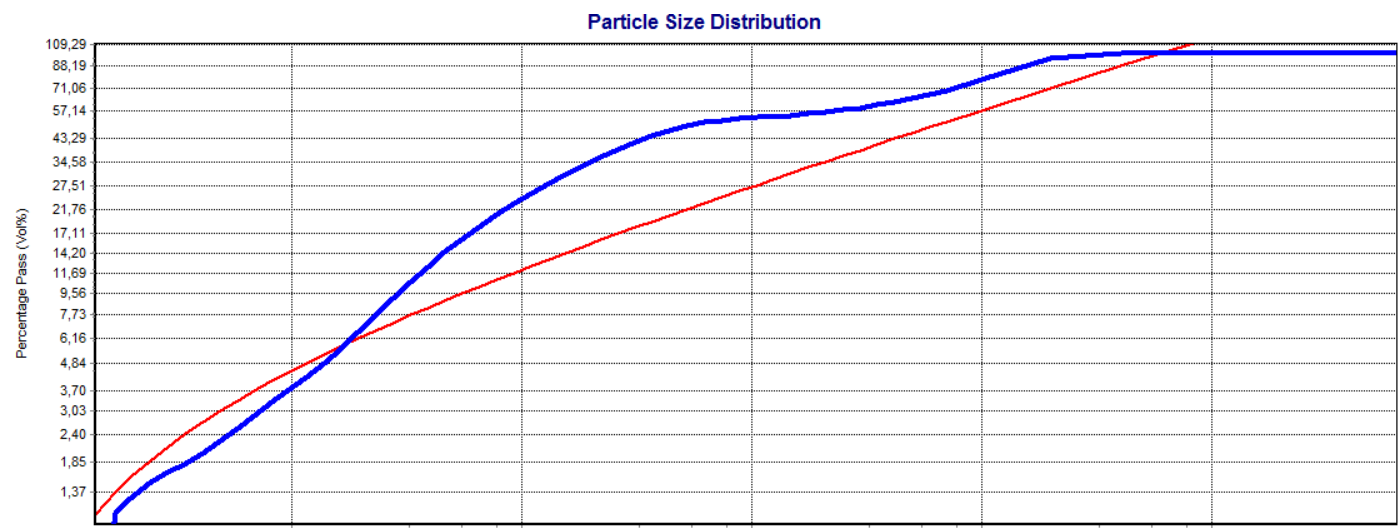

Figura 6 - Curva granulométrica do traço estudado, formulado com q=0,37

Aplicação do software EMMA na análise do empacotamento máximo de partículas utilizando o Metacaulim. 
Observa-se nas simulações dos traços que, segundo TEIXEIRA E CORDON, quanto menor for o valor do coeficiente de empacotamento usado, maior será a quantidade de partículas finas $(<1 \mu \mathrm{m})$ necessárias para aproximar ao máximo a curva de distribuição granulométrica real da mistura. Logo, torna-se difícil alcançar o empacotamento ideal utilizando valores baixos do coeficiente $q$, tornando necessária uma quantidade maior de metacaulim, o material mais fino utilizado nas simulações da mistura, que se trata de um aditivo cimentício. Portanto, quanto maior a quantidade de metacaulim utilizada, maior será a quantidade de cimento necessária na mistura, aumentando, então, seu consumo para misturas dosadas com coeficientes com valores baixos, para que dessa forma possa haver uma aproximação da curva teórica do modelo utilizado, (QUEIROZ).

Os resultados obtidos nestas simulações, mostraram que foi possível na figura 4 otimizar a distribuição granulométrica da mistura. Tal fato deve-se ao coeficiente de empacotamento utilizado na sua dosagem $(q=0,25)$, o qual proporciona a distribuição granulométrica que permite o máximo empacotamento, segundo a literatura. No mais, pode-se ater que dentre os traços simulados, este foi o que mais se aproximou da curva granulométrica ideal, devido a maior facilidade de ajuste da curva pela necessidade de uma maior quantidade de finos (Metacaulim). Portanto, este traço apresenta a maior densidade de empacotamento entre as simulações feitas e estudadas.

\section{Conclusão}

Atualmente contamos com a tecnologia de simulação e produção de concretos em constate evolução, e com o auxílio do EMMA, sua gama de ferramentas e indicadores baseados em parâmetros fidedignos acaba contribuindo bastante no estudo e análise de agregados que podem ser inseridos na mistura do concreto, tornando assim o processo mais confiável. O presente artigo mostra que a simulação de agregados em prol de uma mistura homógena e granulometricamente bem distribuída é possível.

Depois de muitas simulações usando a Areia, Metacaulim e Cimento CP ARI foi encontrado o melhor ajuste em suas quantidades e densidades para que pudesse se ajustar melhor aos coeficientes testados durantes as simulações, trazendo confiabilidade para a produção do concreto usando os agregados presentes nos testes.

\section{Referências Bibliográficas}

AMBROZEWICZ, P. H. L. Materiais de Construção. São Paulo: Pini, 2012. 459 p.

ASSOCIAÇÃO BRASILEIRA DE NORMAS TÉCNICAS. Agregados para Concreto - Especificações: NBR 7211 . Rio de Janeiro, 2009. 9 p.

CASTRO, A. L. de; PANDOLFELLI, V. C. Revisão: Conceitos de dispersão e empacotamento de partícula para a produção de concretos especiais aplicados na construção civil. Cerâmica. São Paulo, 2009. n. 55. p. 18-32.

I. R. de Oliveira, A. R. Studart, R. G. Pileggi, V. C. Pandolfelli. Dispersão e empacotamento de partículas:

Aplicação do software EMMA na análise do empacotamento máximo de partículas utilizando o Metacaulim. 
princípios e aplicações em processamento cerâmico. São Paulo: Fazendo Arte Editorial, 2000.

QUEIROZ, I.S.Oliveira. "Desenvolvimento de concretos de ultra-alta resistência com uso de materiais disponíveis na região do Norte de Minas Gerais." Belo Horizonte, 2016.

C.S TEIXEIRA, H.C.F CORDON. Estudo da influência do empacotamento de partículas nas propriedades do concreto. Mauá, 2016.

MEHTA, P. K. MONTEIRO J. M. Concreto: Microestrutura, propriedades e materiais. São Paulo, Pini, 1994.

BAUER. Método para dosagem racional do concreto. São Paulo: ABCP, 2012. 